G. Bard Ermentrout • David H. Terman

\title{
Mathematical Foundations of Neuroscience
}

43 Springer 


\section{Contents}

1 The Hodgkin-Huxley Equations ....................................................................... 1

$1.1 \quad$ The Resting Potential ..................................................................... 1

1.2 The Nernst Equation ………......................................................

1.3 The Goldman-Hodgkin—Katz Equation ......................................... 5

1.4 Equivalent Circuits: The Electrical Analogue ................................ 8

1.5 The Membrane Time Constant ................................................... 11

1.6 The Cable Equation ………….................................................. 13

1.7 The Squid Action Potential .......................................................... 16

$1.8 \quad$ Voltage-Gated Channels ............................................................ 18

$1.9 \quad$ Hodgkin-Huxley Model ............................................................ 20

1.10 The Action Potential Revisited .................................................... 25

1.11 Bibliography …..................................................................... 27

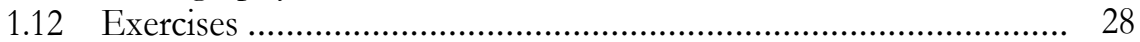

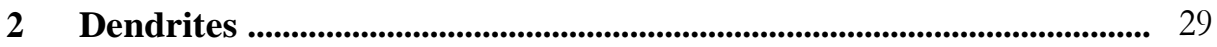

$2.1 \quad$ Multiple Compartments ............................................................ 29

2.2 The Cable Equation ……………............................................... 33

2.3 The Infinite Cable .................................................................. 34

$2.4 \quad$ Finite and Semi-infinite Cables ................................................... 36

2.5 Branching and Equivalent Cylinders ........................................... 38

2.6 An Isolated Junction .................................................................. 40

2.7 Dendrites with Active Processes ................................................... 42

2.8 Concluding Remarks .............................................................. 45

2.9 Bibliography …………….................................................... 45

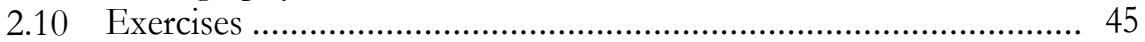

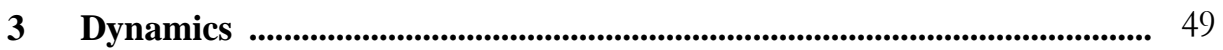

3.1 Introduction to Dynamical Systems ……......................................... 49

3.2 The Morris_Lecar Model ……....................................................... 49

3.3 The Phase Plane ..................................................................... 51

3.3.1 Stability of Fixed Points …………................................ 52

3.3.2 Excitable Systems ………........................................... 53

3.3.3 Oscillations .................................................................. 55 


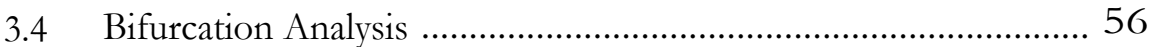

3.4.1 The Hopf Bifurcation ......................................................... 56

3.4.2 Saddle — Node an a Limit Cycle ....................................... 58

3.4.3 Saddle-Homoclinic Bifurcation ....................................... 60

3.4.4 Class I and Class II ..................................................... 62

3.5 Bifurcation Analysis of the Hodgkin-Huxley Equations .................. 63

3.6 Reduction of the Hodgkin - Huxley Model to a Two-Variable Model 66

$3.7 \quad$ FitzHugh-Nagumo Equations ……………….............................. 69

3.8 Bibliography ……………….................................................. 70

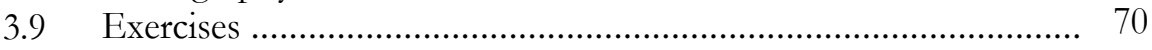

$4 \quad$ The Variety of Channels ................................................................................... 77

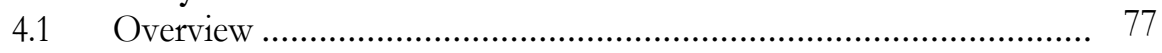

4.2 Sodium Channels .................................................................. 78

4.3 Calcium Channels ...................................................................... 80

4.4 Voltage-Gated Potassium Channels …………............................... 82

4.4.1 A-Current ……………….......................................... 83

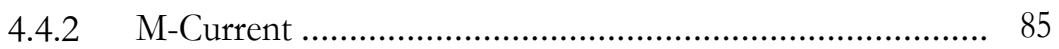

4.4.3 The Inward Rectifier ..................................................... 86

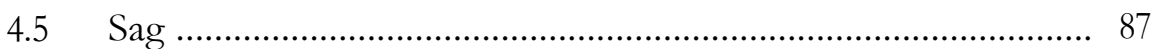

4.6 Currents and lonic Concentrations .............................................. 88

4.7 Calcium-Dependent Channels ...................................................... 90

4.7.1 Calcium Dependent Potassium: The Afterhyperpolarization .............................................. 90

4.7.2 Calcium-Activated Nonspecific Cation Current ................. 93

4.8 Bibliography ........................................................................... 95

4.9 Exercises ………............................................................... 95

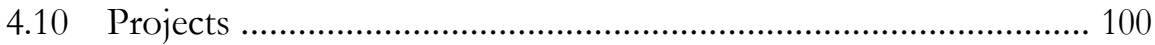

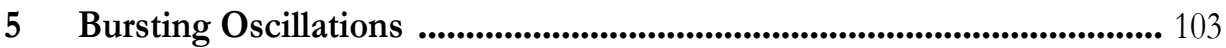

5.1 Introduction to Bursting ............................................................... 103

5.2 Square-Wave Bursters ................................................................... 105

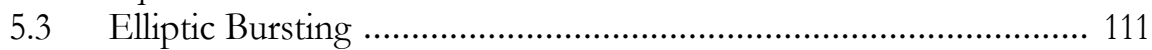

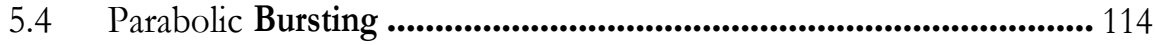

5.5 Classification of Bursters ............................................................. 117

5.6 Chaotic Dynamics .................................................................... 118

5.6.1 Chaos in Square-Wave Bursting Models ........................ 118

5.6.2 Symbolic Dynamics ........................................................ 121

5.6.3 Bistability and the Blue-Sky Catastrophe ........................ 123

5.7 Bibliography 125

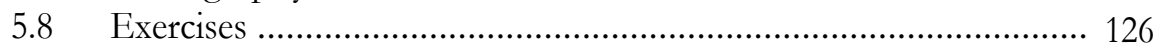


$6 \quad$ Propagating Action Potentials ...................................................... 129

6.1 Traveling Waves and Homoclinic Orbits ................................ 130

6.2 Scalar Bistable Equations ........................................................ 132

6.2.1 Numerical Shooting ................................................... 135

6.3 Singular Construction of Waves ................................................ 136

6.3.1 Wave Trains ................................................................... 139

6.4 Dispersion Relations ............................................................. 139

6.4.1 Dispersion Kinematics ................................................. 141

6.5 Morris—Lecar Revisited and Shilnikov Dynamics .................. 141

6.5.1 Class II Dynamics .................................................... 142

6.5.2 Class I Dynamics ..................................................... 143

6.6 Stability of the Wave ............................................................. 145

6.6.1 Linearization ............................................................... 146

6.6.2 The Evans Function ..................................................... 147

6.7 Myelinated Axons and Discrete Diffusion .............................. 149

6.8 Bibliography ..................................................................... 151

6.9 Exercises ....................................................................... 152

$7 \quad$ Synaptic Channels ............................................................................... 157

7.1 Synaptic Dynamics ............................................................... 158

7.1.1 Glutamate …………………………………….... 161

7.1.2 y-Aminobutyric Acid ..................................................... 162

7.1.3 Gap or Electrical Junctions ............................................ 164

7.2 Short-Term Plasticity ............................................................... 164

7.2.1 Other Models ................................................................. 167

7.3 Long-Term Plasticity ………………………………………...... 168

7.4 Bibliography …………………………………………...... 169

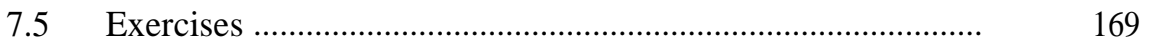

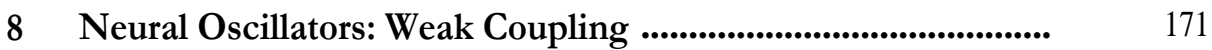

8.1 Neural Oscillators, Phase, and Isochrons ............................... 172

8.1.1 Phase Resetting and Adjoints ................................... 174

8.1.2 The Adjoint ............................................................ 177

8.1.3 Examples of Adjoints ................................................ 178

8.1.4 Bifurcations and Adjoints ......................................... 181

8.1.5 Spike-Time Response Curves ....................................... 186

8.2 Who Cares About Adjoints? .................................................... 187

8.2.1 Relationship of the Adjoint and the Response to Inputs ................................................................. 187

8.2.2 Forced Oscillators …………………………….......... 189

8.2.3 Coupled Oscillators ........................................................ 193

8.2.4 Other Map Models ......................................................... 199

$8.3 \quad$ Weak Coupling …………………………………………........ 202

8.3.1 Geometrie Idea ............................................................ 203

8.3.2 Applications of Weak Coupling ............................... 205 
8.3.3 Synaptic Coupling near Bifurcations ....................... 206

8.3.4 Small Central Pattern Generators ............................ 208

8.3.5 Linear Arrays of Cells ............................................. 213

8.3.6 Two-Dimensional Arrays ………………………........ 217

8.3.7 All-to-All Coupling .................................................. 219

8.4 Pulse-Coupled Networks: Solitary Waves ............................. 223

8.4.1 Integrate-and-Fire Model ......................................... 226

8.4.2 Stability …....................................................... 229

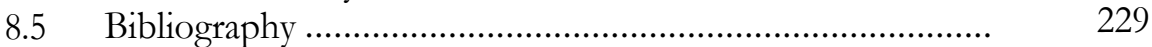

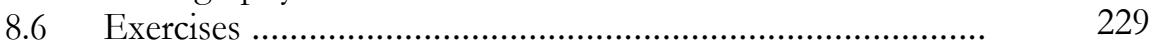

$8.7 \quad$ Projects ..................................................................

$9 \quad$ Neuronal Networks: Fast/Slow Analysis ............................................ 241

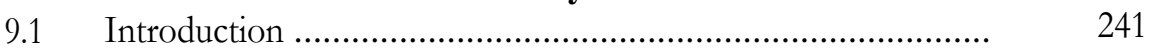

9.2 Mathematical Models for Neuronal Networks ...................... 242

9.2.1 Individual Cells ..................................................... 242

9.2.2 Synaptic Connections ............................................. 243

9.2.3 Network Architecture ...........................................

9.3 Examples of Firing Patterns ................................................. 246

9.4 Singular Construction of the Action Potential ..................... 249

9.5 Synchrony with Excitatory Synapses .................................... 254

9.6 Postinhibitory Rebound ........................................................ 258

9.6.1 Two Mutually Coupled Cells ................................... 258

9.6.2 Clustering ..........................................................

9.6.3 Dynamic Clustering ............................................. 260

9.7 Antiphase Oscillations with Excitatory Synapses ................ 262

9.7.1 Existence of Antiphase Oscillations ........................ 263

9.7.2 Stability of Antiphase Oscillations ......................... 266

9.8 Almost-Synchronous Solutions ........................................... 269

9.8.1 Almost Synchrony with Inhibitory Synapses ......... 269

9.8.2 Almost Synchrony with Excitatory Synapses ......... 271

9.8.3 Synchrony with Inhibitory Synapses ....................... 274

9.9 Slow Inhibitory Synapses ……………………...................... 275

9.9.1 Fast/Slow Decomposition ....................................... 275

9.9.2 Antiphase Solution ............................................... 276

9.9.3 Suppressed Solutions ............................................... 278

9.10 Propagating Waves ………………................................... 278

9.11 Bibliography …….......................................................... 282

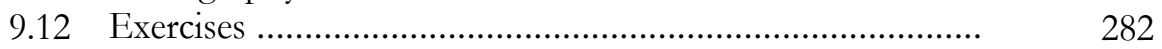

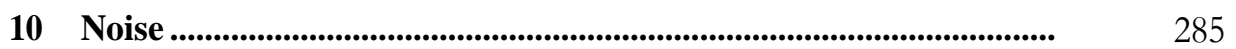

10.1 Stochastic Differential Equations ....................................... 287

10.1.1 The Wiener Process ................................................ 288

10.1.2 Stochastic Integrals ................................................ 289

10.1.3 Change of Variables: Itö's Formula ........................ 289 
10.1.4 Fokker-Planck Equation: General Considerations ..... $\quad 290$

10.1.5 Scalar with Constant Noise .................................... 293

10.1.6 First Passage Times ............................................... 295

10.2 Firing Rates of Scalar Neuron Models ................................. 299

10.2.1 The Fokker-Planck Equation .................................. 299

10.2.2 First Passage Times ............................................... 303

10.2.3 Interspike Intervals ......................................... 306

10.2.4 Colored Noise ..................................................... 307

10.2.5 Nonconstant Inputs and Filtering Properties ............. 309

10.3 Weak Noise and Moment Expansions ................................ 310

10.4 Poisson Processes ....................................................... 314

10.4.1 Basic Statistics .................................................... 314

10.4.2 Channel Simulations .......................................... 317

10.4.3 Stochastic Spike Models: Beyond Poisson ............... 319

10.5 Bibliography ......................................................... 321

10.6 Exercises .................................................................... 321

10.7 Projects .................................................................... 326

11 Firing Rate Models ......................................................................... 331

11.1 A Number of Derivations ............................................. 332

11.1.1 Heuristic Derivation ............................................ 332

11.1.2 Derivation from Averaging .................................. 336

11.1.3 Populations of Neurons ...................................... 338

11.2 Population Density Methods .......................................... 341

11.3 The Wilson-Cowan Equations ........................................ 344

11.3.1 Scalar Recurrent Model ........................................ 346

11.3.2 Two-Population Networks .................................. 346

11.3.3 Excitatory_-Inhibitory Pairs ............................... 350

11.3.4 Generalizations of Firing Rate Models ................... 356

11.3.5 Beyond Mean Field ............................................ 359

11.4 Some Methods for Delay Equations .................................. 361

11.5 Exercises ..................................................................... 363

11.6 Projects .................................................................... 365

12 Spatially Distributed Networks $\quad$............................................. 369

12.1 Introduction .......................................................... 369

12.2 Unstructured Networks ................................................. 370

12.2.1 McCulloch-Pitts .............................................. 370

12.2.2 Hopfield's Model ............................................... 371

12.2.3 Designing Memories ........................................... 373

12.3 Waves ......................................................................... 375

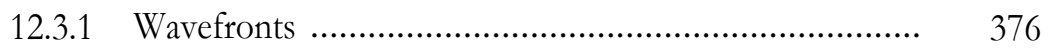

12.3.2 Pulses ............................................................... 379 


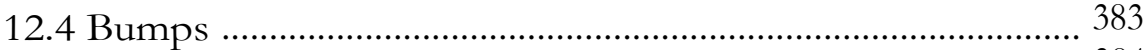

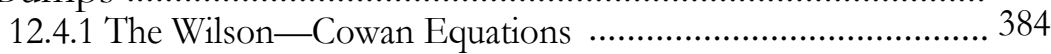

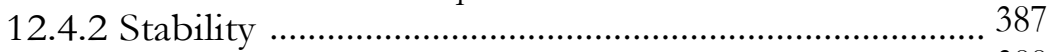

12.4.3 More General Stability .................................................. 388

12.4.4 More General Firing Rates ............................................. 389

12.4.5 Applications of Bumps ................................................... 390

12.5 Spatial Patterns: Hallucinations ................................................... 394

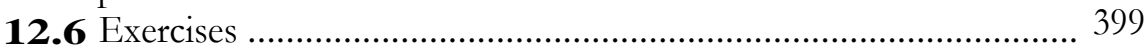

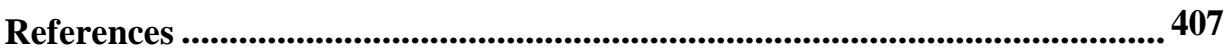

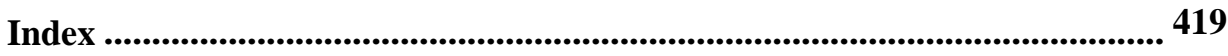

